\title{
Gestational Methylazoxymethanol Exposure Leads to NMDAR Dysfunction in Hippocampus During Early Development and Lasting Deficits in Learning
}

\author{
Melissa A Snyder', Alicia E Adelman' and Wen-Jun Gao*,' \\ 'Department of Neurobiology and Anatomy, Drexel University College of Medicine, Philadelphia, PA, USA
}

\begin{abstract}
The N-methyl-D-aspartate (NMDA) receptor has long been associated with learning and memory processes as well as diseased states, particularly in schizophrenia (SZ). Additionally, SZ is increasingly recognized as a neurodevelopmental disorder with cognitive impairments often preceding the onset of psychosis. However, the cause of these cognitive deficits and what initiates the pathological process is unknown. Growing evidence has implicated the glutamate system and, in particular, N-methyl-D-aspartate receptor (NMDAR) dysfunction in the pathophysiology of SZ. Yet, the vast majority of SZ-related research has focused on NMDAR function in adults leaving the role of NMDARs during development uncharacterized. We used the prenatal methylazoxymethanol acetate (MAM, EI7) exposure model to determine the alterations of NMDAR protein levels and function, as well as associated cognitive deficits during development. We found that MAM-exposed animals have significantly altered NMDAR protein levels and function in the juvenile and adolescent hippocampus. Furthermore, these changes are associated with learning and memory deficits in the Morris Water Maze. Thus, in the prenatal MAM-exposure SZ model, NMDAR expression and function is altered during the critical period of hippocampal development. These changes may be involved in disease initiation and cognitive impairment in the early stage of SZ. Neuropsychopharmacology (20I3) 38, 328-340; doi:I0.1038/npp.20 I2. I80; published online I2 September 2012
\end{abstract}

Keywords: neurodevelopment; glutamate; hippocampus; schizophrenia; animal model

\section{INTRODUCTION}

Schizophrenia (SZ) is a devastating mental illness that is now recognized as a neurodevelopmental disorder that begins in prenatal or perinatal life. Evidence suggests that genetic susceptibilities interact with environmental factors to alter the neurodevelopmental trajectory, resulting in psychosis in the second or third decade of life (Lewis and Levitt, 2002). While it remains unknown how neurodevelopment is altered, many brain regions are implicated in SZ. This includes the hippocampus, which is critical for learning and memory, and is consistently found to be altered in human schizophrenics, both in terms of anatomy and function (Harrison, 2004). Furthermore, hippocampal changes are present in prodromal and first-episode schizophrenics, suggesting that they are not secondary to the illness or treatment (Bogerts et al, 1990; Grace, 2012; Medoff et al, 2001; Witthaus et al, 2009). In addition, the glutamate system and, in particular, $N$-methyl-D-aspartate receptors (NMDARs) within the hippocampus and cortex are

*Correspondence: Dr W-J Gao, Department of Neurobiology and Anatomy, Drexel University College of Medicine, 2900 Queen Lane, Philadelphia, PA 19129, USA, Tel: +215 991 8907, Fax: +215 843 9802, E-mail: wgao@drexelmed.edu

Received 24 May 2012; revised 10 August 2012; accepted 15 August 2012 important for disease symptoms and the progression of SZ (Hradetzky et al, 2012; Kantrowitz and Javitt, 2010).

NMDARs are complexes formed by an NR1 subunit with NR2A-D and/or NR3A-B subunits to form an operational channel. Altering NMDAR subunit composition can have important consequences both for channel function and cognitive processing (Tang et al, 1999; von Engelhardt et al, 2008; Zhao et al, 2005). In hippocampus, like some cortical regions, NMDAR subunit expression is developmentally regulated and preventing the switch of NMDARs to their mature form can severely impact synaptogenesis and synaptic maturation (Brigman et al, 2010; Gambrill and Barria, 2011; Gray et al, 2011; Roberts et al, 2009). Therefore, misregulation of NMDAR subunit composition and function during hippocampal development may contribute to the pathogenesis in SZ. There is a vast literature examining NMDARs in association with SZ; however, the majority focused on adult animals (Coyle, 2006) or postmortem human tissues (Meador-Woodruff et al, 2003), leaving the possible role of NMDARs in early postnatal development uncharacterized. To determine whether developmental alterations of NMDAR expression and function could contribute to hippocampal changes in neurodevelopment and SZ, we used the well-characterized prenatal methalozoxymethanol acetate (MAM) exposure model of SZ (Featherstone et al, 2009; Flagstad et al, 2004; Goto and 
Grace, 2006; Lodge and Grace, 2007; Moore et al, 2006). Adult offspring exposed to MAM on gestational day 17 have deficits similar to those seen in schizophrenics, including altered activity in the hippocampus (Lodge and Grace, 2007) and a similar neuroanatomical profile (Moore et al, 2006). Further, they exhibit behavioral changes that mimic aspects of the positive, negative, and cognitive symptoms of SZ, including an increased response to PCP and amphetamine (Flagstad et al, 2004; Moore et al, 2006), social impairment (Le Pen et al, 2006), and deficits in working memory (Gourevitch et al, 2004). Together, the deficits induced by prenatal MAM exposure closely parallels disease progression in human SZ. Therefore, understanding how MAM exposure alters NMDAR expression and function could provide insight into how hippocampal development is altered in SZ. In this study, we find that MAM exposure significantly alters NMDAR protein levels and function in the juvenile hippocampus, which is correlated with a deficit in long-term potentiation (LTP) and performance on the Morris water maze (MWM). Further, the changes to NMDARs persist into adolescence, and thus may represent a mis-step in development that is involved in disease initiation and cognitive impairment in the early stage of SZ.

\section{MATERIALS AND METHODS}

\section{Animals}

All animal procedures were performed in accordance with the National Institutes of Health Guide for the Care and Use of Laboratory Animals and were approved by the Drexel University College of Medicine Animal Care and Use Committee. Thirty-two adult pregnant Sprague-Dawley rats were purchased from Charles River Laboratories International (Wilmington, MA) and were intraperitoneally (I.P.) injected with $25 \mathrm{mg} / \mathrm{kg}$ MAM or saline vehicle control on gestational day 17 (E17). Pups were usually born on embryonic day 21 and were weaned and rehoused on postnatal day 21. For all experiments, animals aged P7 were classified as neonatal, P12-21 as juveniles, P28-45 as adolescents based on others' (Spear, 2000) and our previous studies (Wang and Gao, 2009, 2010).

\section{Tissue Collection and Western Blot}

Animals were perfused with ice-cold sucrose buffer (in mM: 320 sucrose, 4 HEPES-NaOH buffer, pH 7.4, 2 EGTA, 1 sodium orthovanadate, 0.1 phenylmethylsulfonyl fluoride, 50 sodium fluoride, 10 sodium pyrophosphate, 20 glycerophosphate, with $1 \mu \mathrm{g} / \mathrm{ml}$ leupeptin and $1 \mu \mathrm{g} / \mathrm{ml}$ aprotinin). The hippocampus was dissected, homogenized in sucrose buffer, and then centrifuged at $1000 \mathrm{~g}$ for $10 \mathrm{~min}$ to remove large cell fragments and nuclear materials. The resulting supernatant was centrifuged at $17000 \mathrm{~g}$ for $15 \mathrm{~min}$ to yield cytoplasmic proteins in the supernatant. The pellet from this spin was resuspended in homogenization buffer and centrifuged at $17000 \mathrm{~g}$ for an additional $15 \mathrm{~min}$ to yield washed synaptosomes. The synaptosomal fraction then was hypoosmotically lysed and centrifuged at $25000 \mathrm{~g}$ for $20 \mathrm{~min}$ to yield synaptosomal plasma membranes in the pellet. A bicinchoninic acid protein assay was performed to determine protein concentration. The protein sample was mixed with laemmli sample buffer, boiled for $5 \mathrm{~min}$, and separated on a 7.5\% SDS-PAGE gel. After electrophoresis, proteins were transferred to polyvinylidene difluoride membranes (Millipore). The membrane was blocked in 5\% nonfat milk and probed with primary antiserum. Each blot was simultaneously probed for anti-rabbit NR2A (Millipore, 04-901, $1: 4000$ ), anti-rabbit NR3A (Millipore, 07-356, 1:4000), and anti-rabbit NR3B (Tocris, 2060, 1:4000), then stripped with Restore Western Blot Stripping Buffer (Thermo Scientific) and reprobed for anti-mouse NR1 (Invitrogen, 32-5000, $1: 5000$ ), anti-mouse NR2B (Millipore, 05-920, 1:2000), and anti-mouse actin (Sigma, A5316, 1:100000), which was used as a loading control. The blots were incubated with horseradish peroxidase-coupled anti-rabbit or anti-mouse IgG secondary antibody (Vector Laboratories), and proteins were visualized using enhanced chemiluminescence (ECL Plus, Amersham Biosciences). Protein expression for each subunit was evaluated by densitometry using Image-Pro Plus software. Additionally, samples from each animal were run at least four times to minimize interblot variance. Data were analyzed statistically using Student's $t$-test and were presented as mean $\pm \mathrm{SE}$.

\section{Electrophysiology}

Experiments were conducted as previously described (Wang and Gao, 2010). Juvenile (aged P11-23) and adolescent (aged P29-39) saline- and MAM-exposed animals of either sex were used. Briefly, animals were anesthetized with euthasol $(0.2 \mathrm{ml} / \mathrm{kg}$, I.P. $)$ and perfused with ice-cold, oxygenated artificial cerebrospinal fluid (ACSF, in mM: $124 \mathrm{NaCl}, 2.5$ $\mathrm{KCl}, 1.25 \mathrm{NaH}_{2} \mathrm{PO}_{4}, 2 \mathrm{CaCl}_{2}, 1 \mathrm{MgSO}_{4}, 26 \mathrm{NaHCO}_{3}$, and 10 dextrose, $\mathrm{pH}$ 7.4). The brains were removed and dorsal hippocampal slices were cut $(300 \mu \mathrm{m})$ into an ice-cold bath of oxygenated ACSF using a vibrotome tissue slicer (Leica Microsystems). Slices were transferred to a holding chamber, submerged in oxygenated ACSF at $35^{\circ} \mathrm{C}$ for $1 \mathrm{~h}$ and then remained at room temperature until used for recording. Slices were placed into a recording chamber mounted on an Olympus upright microscope (BX51), where they were bathed in oxygenated ACSF. Neurons were visualized with infrared differential interference video microscopy. To record AMPAR- and NMDAR-mediated currents, somatic whole-cell voltage-clamp recordings were obtained from CA1 pyramidal cells using patch electrodes with an open tip resistance of 5-7 M $\Omega$ (internal solution, in $\mathrm{mM}: 110 \mathrm{D}-$ gluconic acid, $110 \mathrm{CsOH}, 10 \mathrm{CsCl}_{2}, 1 \mathrm{EGTA}, 1 \mathrm{CaCl}_{2}, 5 \mathrm{QX}-$ 314, 1 ATP-Mg, 10 HEPES, at $\mathrm{pH} 7.3$, adjusted with $\mathrm{CsOH}$ ). A stimulating electrode was placed in the middle of the stratum radiatum $150-250 \mu \mathrm{m}$ from the soma of the recorded cell. Synaptically evoked excitatory postsynaptic currents (EPSCs) were evoked by a bipolar electrode using paired pulses (100 ms interstimulus interval) and recorded first at a holding potential of $-60 \mathrm{mV}$ and then $+60 \mathrm{mV}$ in ACSF containing the $\mathrm{GABA}_{\mathrm{A}}$ receptor antagonist picrotoxin (50-100 $\mu \mathrm{M}$, Tocris).

In a separate set of experiments, the patch pipette was filled with a potassium gluconate internal solution (in $\mathrm{mM}$ : 120 potassium gluconate, $20 \mathrm{KCl}, 4$ ATP-Na, $0.3 \mathrm{Na}_{2} \mathrm{GTP}$, 5 Na-phosphocreatine, 0.1 EGTA, 10 HEPES, pH 7.3, $305 \mathrm{mosmol} / \mathrm{l})$. Whole-cell current clamp was used to record action potentials (APs) in response to varying step currents 
from -300 to $+400 \mathrm{pA}$ with $50 \mathrm{pA}$ increment. The recording was then switched into voltage-clamp mode with membrane potentials held at $-70 \mathrm{mV}$ in the presence of picrotoxin $(100 \mu \mathrm{M})$ to record spontaneous EPSCs (sEPSCs), or with both picrotoxin and tetrodotoxin (TTX, $0.5 \mu \mathrm{M})$ for miniature EPSCs (mEPSCs).

Extracellular recording. For LTP, experiments were conducted in the stratum radiatum of the CA1 hippocampus. The recording electrode (1-3 M $\Omega$ tip resistance) was filled with ACSF (see above) and placed $\sim 500 \mu \mathrm{m}$ away from the stimulating electrode. Hippocampal slices were prepared as above. For baseline recording, the stimulus intensity was set to elicit a field potential of $\sim 0.5 \mathrm{mV}$ peak amplitude. There was no statistical difference in the current intensity used between saline and MAM slices $(p=0.19)$. Stimulation was given every $30 \mathrm{~s}$ for $20 \mathrm{~min}$ to generate baseline data. Only recordings with stable baselines were used for LTP recording. To induce LTP, slices were stimulated with high-frequency stimulation $(1 \mathrm{~s}, 100 \mathrm{~Hz}$ train, repeated two times at $30 \mathrm{~s}$ intervals). Thereafter, stimulation was given every $30 \mathrm{~s}$ for further recording of $40 \mathrm{~min}$.

Data analysis. All experiments were conducted with Axon MultiClamp 700B amplifier (Molecular Devices), and data were acquired using pCLAMP 9.2 software and analyzed using Clampfit 9.2 (Molecular Devices). A typical s/mEPSC was selected to create a sample template for the event detections within a 5-min data period. The frequency (number of events) and amplitude of the individual events were examined with the threshold set at the medium level (ie, 5) in Clampfit. The detected events were then visually inspected to ensure specificity. The amplitudes of the evoked EPSCs were measured by averaging 30 traces from the onset to the peak of the EPSC. Because we calculated the relative ratio, NMDA current was measured $50 \mathrm{~ms}$ after the peak in evoked EPSCs recorded at $+60 \mathrm{mV}$ in the presence of picrotoxin $(50-100 \mu \mathrm{M})$. This analysis avoids the AMPA receptor-mediated current, which usually returns to baseline within $50 \mathrm{~ms}$. Only the neurons that produced stable baseline EPSCs without rundown were used for further analysis. To determine whether the recordings were stable and reliable, the baseline EPSC amplitudes of each neuron were exported to excel to make a scatter graph and examined the linear relationship by adding a trend line. In addition, the input resistance of each neuron was monitored by injecting negative current $(-100 \mathrm{pA}, 200 \mathrm{~ms})$ $500 \mathrm{~ms}$ prior to EPSC induction during recording. The stable baseline recording was thus determined by a recording showing no progressive changes (increase or decrease) in EPSC amplitudes, including a flat/horizontal trend line and a small $R^{2}$ value, as well as no alteration in input resistance. All neurons without stable baseline recording of EPSCs and with input resistance increased more than 20\% were discarded for further analysis. For paired-pulse data, 30 traces in a run were averaged and paired-pulse ratio was determined as the peak amplitude of EPSC2/EPSC1. The APs recorded in current-clamp mode were used to measure the resting membrane potential, input resistance, AP threshold, AP half-width, peak AP amplitude, and size of afterhyperpolarization. For LTP experiments, the slope of the field potential was averaged for baseline recording (criteria used for stable baseline are same as aforementioned EPSC recording) and compared with the average slope of the EPSPs in the first 2 min of post-tetanus stimulation or the average slope of the EPSPs in the last $2 \mathrm{~min}$ of post-tetanus stimulation. All data were analyzed with Student's $t$-test for statistical significance with $95 \%$ confidence, and were presented as mean \pm SE. The majority of experiments were conducted in male rats; however, female data were included in some experiments and no significant sex difference was found.

\section{Morris Water Maze}

Spatial learning in male saline and MAM rats was assessed using the MWM beginning at age P17 (juvenile) or at P40 (adolescent). The animals were required to locate a hidden platform located in a fixed position using visual cues. Each rat was trained over 5 consecutive days, with four trials each day, and allotted a maximum of $60 \mathrm{~s}$ to locate the submerged platform in a 1-m diameter water maze. The average of the four trials was used as the average latency to reach the platform on each training day. A repeated measures ANOVA followed by post-hoc analysis was used to determine statistical significance. A probe memory testing occurred $24 \mathrm{~h}$ after the last training session and was used to evaluate the animals' ability to recall the learned platform location. With the platform removed, each animal was given $60 \mathrm{~s}$ to swim while being tracked with a computerized video system. The amount of time spent swimming in the previous platform location quadrant minus the time spent swimming along the pool edge was calculated and compared between saline and MAM animals. The total amount of time spent swimming along the pool edge was also compared between saline and MAM animals. These data were analyzed statistically using Student's $t$-test. Following training and probe trials, the latency to find a visible platform was recorded. Swim speed was calculated by taking the total distance traveled divided by the trial length $(60 \mathrm{~s})$.

\section{RESULTS}

\section{NMDAR Protein Levels are Reduced in the Hippocampus of Juvenile MAM-Exposed Rats}

To determine whether NMDARs are altered during development in a SZ model, we first examined how prenatal MAM exposure affects NMDAR protein levels in different age groups, including neonatal (P7), juvenile (P14 and P21), and adolescent (P45) animals. We collected synaptic plasma membrane fractions from saline and MAM animals, and probed for NMDAR subunits, including NR1, NR2A, NR2B, NR3A, and NR3B. Actin was used as a loading control. We found no significant difference in NR1, NR2A, NR2B, or NR3A subunit levels at P7 (Figure $1 \mathrm{a}, p=0.25,0.45,0.32$, $0.72,0.55$, respectively, $n=7)$. NR3B was not thoroughly examined in this age group, because NR3B protein levels are extremely low at this developmental time point and thus not reliably detectable by western blot (Henson et al, 2010).

However, at P14, there was a significant decrease in NR3A in the hippocampus of MAM animals compared with saline (Figure 1b, NR3A saline $1.02 \pm 0.03$, MAM $0.841 \pm 0.04$, 
a
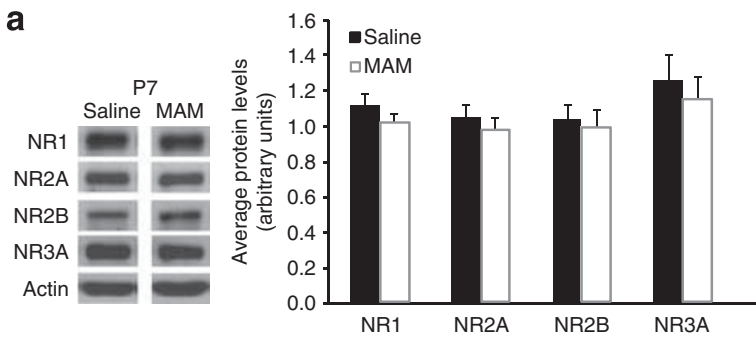

b
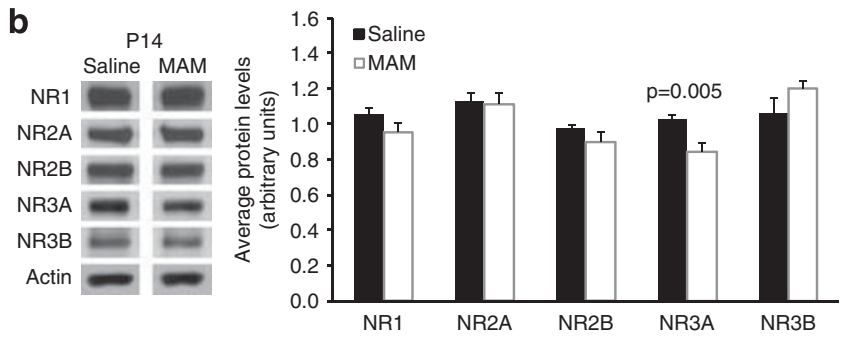

C
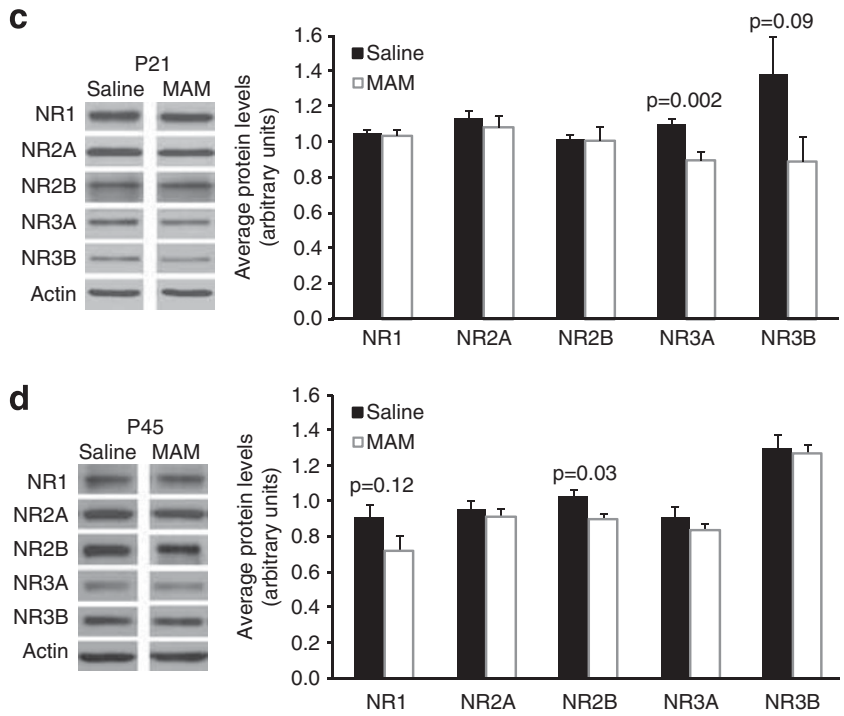

Figure I NMDAR protein levels are reduced in the hippocampus of juvenile MAM-exposed rats. (a-d), Representative western blots and summary histograms show the NMDAR subunit protein levels in synaptic plasma membrane fractions from saline- and MAM-exposed animals at P7 (a), PI 4 (b), P2I (c), and P45 (d). No significant difference was found for any subunit at P7 (Note: NR3B was not detectable at P7). However, MAM exposure results in a significant decrease in NR3A at PI4 ( $n=8$ for both saline and MAM, $p=0.005$ ), and at P2I (saline, $n=7, M A M, n=9$, $p=0.002$ ) compared to saline. By $P 45$, there are no longer significant differences in NR3A, yet, there is a significant decrease in NR2B $(n=8$, $p=0.03)$.

$p=0.005, n=8)$. No difference at P14 was found for NR1, $\mathrm{NR} 2 \mathrm{~A}, \mathrm{NR} 2 \mathrm{~B}$, and NR3B $(p=0.21,0.81,0.22$, and 0.17 , respectively, $n=8$ ). Furthermore, at P21, MAM animals had significantly lower NR3A and a trend toward lower NR3B protein levels (Figure 1c, NR3A saline 1.08 \pm 0.03 , MAM $0.882 \pm 0.05, p=0.002, n=7$ and 8 , respectively; NR3B saline 1.362 \pm 0.21 , MAM $0.875 \pm 0.14, p=0.09, n=4$ and 5 , respectively). Interestingly, in the adolescent (P45), we found no difference between saline and MAM animals for NR1, NR2A, NR3A, and NR3B subunits (Figure 1d, NR1 $p=0.12$, NR2A $p=0.51$, NR3A $p=0.30$, saline, $n=7$, MAM, $n=9$; and NR3B $p=0.77, n=5$ ), but a significant decrease in NR2B in MAM animals (NR2B saline 1.02 \pm 0.04 , MAM $0.891 \pm 0.03, p=0.03$, saline, $n=7, \mathrm{MAM}, n=9)$. Together, these data indicate that prenatal MAM exposure alters NMDAR protein levels during a critical time period for the developing hippocampus.

\section{NMDA-EPSCs are Increased in Juvenile MAM-Exposed Rats}

We hypothesized that the MAM-induced alteration in NMDAR protein levels would affect the function of NMDARs at synapses. We first determined whether prenatal MAM exposure had an effect on CA1 pyramidal neuron health. We used whole-cell patch clamp recording in current-clamp mode to record APs, in slices from juvenile (P13-20) and adolescent (P29-39) animals in response to varying step currents from $-300 \mathrm{pA}$ to $+400 \mathrm{pA}$. MAM exposure had no effect on resting membrane potential, AP threshold, AP peak amplitude, AP 1/2 width, $20-80 \%$ rise time, and the afterhyperpolarization in juveniles or adolescents (Table 1). However, we found an increase in spike number with high current injection $(>300 \mathrm{pA})$ in cells from juvenile MAM animals (Figure $2 \mathrm{a}$, saline $n=6, \operatorname{MAM} n=7, p<0.05$ ). There was no difference in these same measures in adolescence (Figure 2b, saline $n=11$, MAM $n=12, p>0.05$ ). Thus, it appears that MAM does not significantly alter cell health in both age groups.

We next assessed NMDAR function in juvenile and adolescent rats. We recorded EPSCs evoked by paired-pulse stimulation of stratum radiatum in voltage-clamp mode in the presence of picrotoxin, with first evoking currents between $50-100 \mathrm{pA}$ in a holding potential of $-60 \mathrm{mV}$. In the same cell we then recorded evoked EPSCs with a holding potential of $+60 \mathrm{mV}$ (Figure $3 \mathrm{a}_{1}$ ). This allowed us to compare the AMPA-EPSC amplitude, the NMDA-EPSC amplitude, the NMDA/AMPA ratio, and to calculate pairedpulse ratio, which may indicate presynaptic function, between saline and MAM animals. We found no differences in the AMPA-EPSC amplitude (Figure $3 b_{1}$ ) and in pairedpulse ratio (PPR AMPA-EPSC saline 2.06 \pm 0.29 , MAM $1.92 \pm 0.14, p=0.65 ;$ PPR NMDA-EPSC saline $1.06 \pm 0.18$, MAM $0.84 \pm 0.083, p=0.24, n=7$ and 9 , respectively) in the juvenile (P11-19) rat hippocampal neurons. However, cells from juvenile MAM animals showed significantly larger NMDA-EPSC amplitudes (Figure $3 b_{1}$, first pulse: saline $49.3 \pm 6.14 \mathrm{pA}, \mathrm{MAM} 149.3 \pm 35.0, p=0.026$; second pulse: saline $46.4 \pm 4.8 \mathrm{pA}, \mathrm{MAM} 111.3 \pm 21.9 \mathrm{pA}, p=0.022 ; n=7$ and 9, respectively), as well as a larger NMDA/AMPA ratio (Figure $3 c_{1}$, first pulse: saline $0.88 \pm 0.13$, MAM $1.94 \pm 0.42$, $p=0.04$; second pulse: saline $0.46 \pm 0.08$, MAM $0.75 \pm 0.11$, $p=0.045, n=7$ and 9 , respectively), indicating that juvenile MAM animals had increased NMDAR current at synapses. This result is consistent with reduced NR3A protein levels in synaptic plasma membrane fractions (Figure 1), because NR3A is a known negative regulator of NMDAR current (Das et al, 1998; Perez-Otano et al, 2001; Sasaki et al, 2002; Tong et al, 2008). Thus, MAM-exposed animals had significantly altered glutamatergic signaling in juvenile rat hippocampal neurons.

It is possible that the changes of NMDAR function persist throughout development. Alternatively, the changes in juvenile animals may represent a transient development 
Table I Physiological Properties of CAI Pyramidal Neurons in Saline and MAM Animals

\begin{tabular}{|c|c|c|c|c|c|c|}
\hline & $\begin{array}{l}\text { Resting membrane } \\
\text { potential }(\mathrm{mV})\end{array}$ & $\begin{array}{l}\text { Spike threshold } \\
(\mathrm{mV})\end{array}$ & $\begin{array}{c}\text { Peak spike } \\
\text { amplitude }(\mathrm{mV})\end{array}$ & $\begin{array}{c}\text { Afterhyperpolarization } \\
(\mathrm{mV})\end{array}$ & $\begin{array}{l}\text { Spike half-width } \\
\text { (ms) }\end{array}$ & $\begin{array}{c}20-80 \% \text { rise time } \\
(\mathrm{ms})\end{array}$ \\
\hline Juvenile saline & $-66.1 \pm 2.0$ & $-41.7 \pm 3.1$ & $77.3 \pm 2.9$ & $-4.7 \pm 1.1$ & $1.5 \pm 0.1 \mid$ & $0.31 \pm 0.02$ \\
\hline Adolescent saline & $-66.2 \pm 1.6$ & $-40.3 \pm 2.4$ & $81.6 \pm 3.9$ & $-61 \pm 0.86$ & $1.3 \pm 0.17$ & $0.28 \pm 0.02$ \\
\hline Adolescent MAM & $-64.7 \pm 1.9$ & $-39.9 \pm 2.6$ & $81.5 \pm 2.8$ & $-7.3 \pm 1.2$ & $1.2 \pm 0.07$ & $0.27 \pm 0.01$ \\
\hline
\end{tabular}

There were no significant differences between cells from saline and MAM animals in both ages (all $p>0.05$ ).

$\mathbf{a}_{1}$

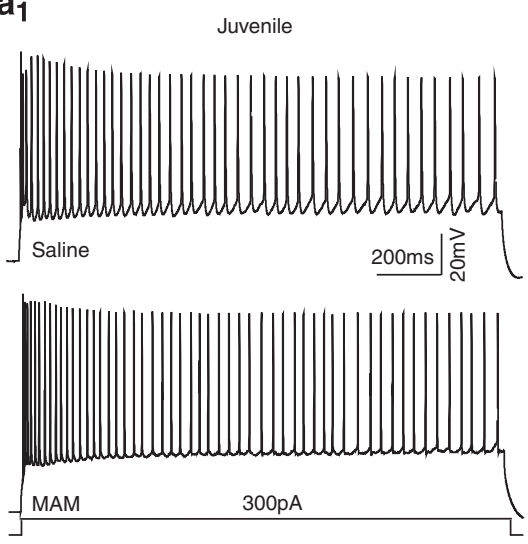

b1

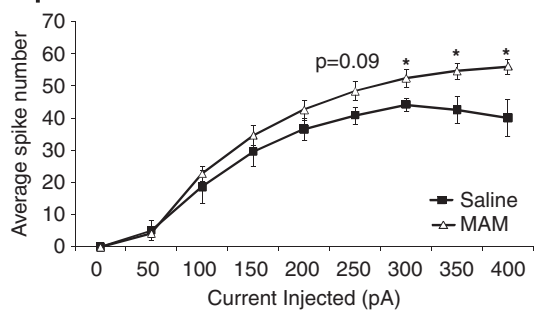

$\mathbf{a}_{2}$
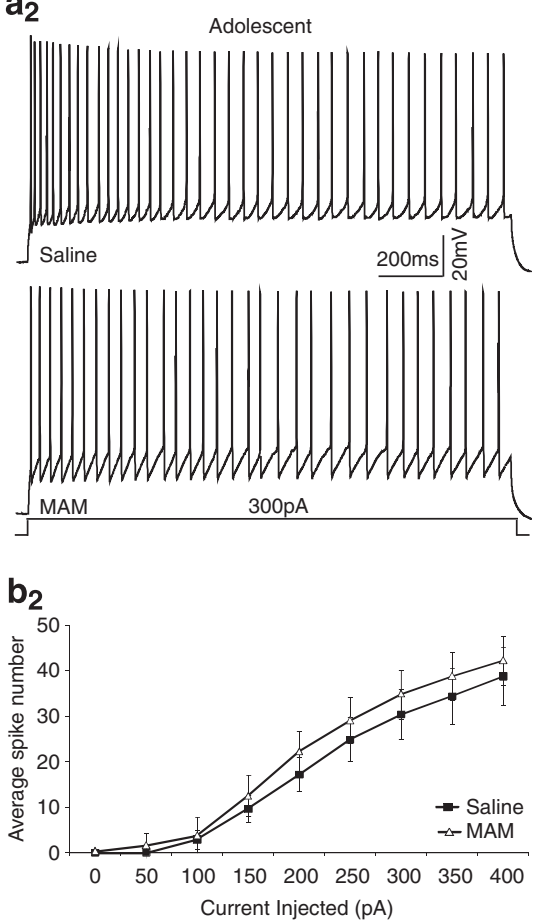

Figure 2 MAM exposure slightly increases excitability of CAI pyramidal neurons in hippocampus in juvenile animals. Representative action potentials (a and $\left.a_{2}\right)$ and summary graphs $\left(b_{1}\right.$ and $\left.b_{2}\right)$ from juvenile and adolescent saline and MAM-exposed animals. $\left(a_{1}\right.$ and $\left.b_{1}\right)$ Juvenile MAM-exposed animals had an increased spike number only with high current injection compared with saline controls (saline $n=6, \operatorname{MAM} n=7$, $* p<0.05$ ). (a $a_{2}$ and $\left.b_{2}\right)$ There is no difference in neuronal excitability in adolescent animals (saline $n=11$, MAM $n=12, p>0.05$ ).

mis-step that contributes to deficits found in adult MAM animals. Because there was no significant change in NR3A protein levels, but a significant decrease in NR2B protein levels in adolescent MAM animals, we wondered whether NMDAR function in MAM animals was still altered. We reexamined NMDA-EPSC amplitude and the NMDA/AMPA ratio in adolescent rats (P30-39; Figure $3 \mathrm{a}_{2}$ ). Interestingly, we found no difference in NMDA-EPSC amplitudes (Figure $3 b_{2}$, first pulse: saline $72.1 \pm 14.5 \mathrm{pA}, \mathrm{MAM} 77.1 \pm 9.29 \mathrm{pA}, p=0.7$; second pulse: saline $56.44 \pm 7.2 \mathrm{pA}, \mathrm{MAM} 62.5 \pm 7.7 \mathrm{pA}$, $p=0.6 ; n=7$ and 8 , respectively), or the NMDA/AMPA ratio (Figure $3 c_{2}$, first pulse: saline $0.70 \pm 0.11$, MAM $0.73 \pm$ $0.08, p=0.8$, second pulse: saline $0.34 \pm 0.06$, MAM $0.41 \pm$ $0.04, p=0.3 ; n=7$ and 8 , respectively) in MAM-exposed animals compared with saline control. This is consistent with a normal NR3A expression, as well as normal NR1, NR2A, and NR3B protein levels in adolescent animals, supporting a relatively recovered NMDAR function in adolescence. However, we did find a significant decrease in the decay time of the second NMDA-EPSC in MAM animals (saline 91.54 $\pm 4.1 \mathrm{~ms}$, MAM 70.9 $\pm 2.7, p=0.008$, $n=7$ and 8 , respectively). This is consistent with the reduced NR2B protein levels in MAM animals because NR2B-containing NMDARs have a slower decay time than NR2A-containing receptors (Cull-Candy et al, 2001; Wang et al, 2008).

Further, we found a small but significant decrease in paired-pulse ratio specifically for AMPA-EPSCs, but not NMDA-EPSCs (AMPA-EPSC PPR, saline $1.65 \pm 0.09$, MAM $1.42 \pm 0.06, p=0.038$; NMDA-EPSC PPR, saline $0.86 \pm 0.11$, MAM $0.84 \pm 0.07, p=0.8 ; n=7$ and 8 , respectively), although both the first and second AMPA-EPSC amplitudes appeared to be comparable between MAM-exposed rats and saline control ( $p=0.77$ and 0.37 , respectively). This may indicate a change in presynaptic function in adolescent animals. Additionally, given that we experimentally controlled the size of the AMPA-mediated EPSC, it is unclear whether MAM exposure specifically alters presynaptic 

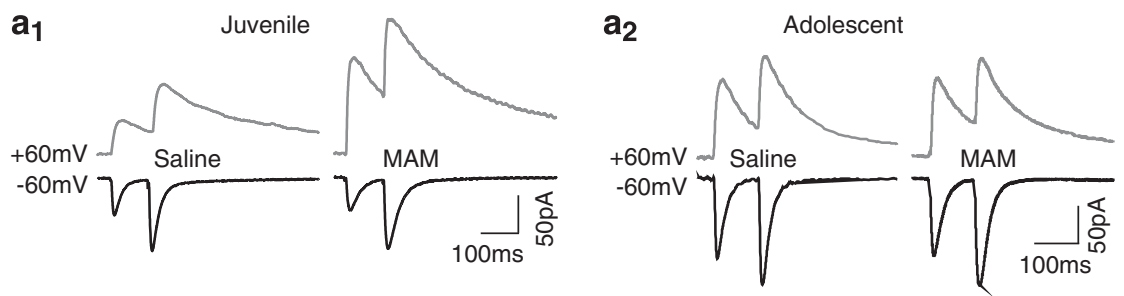

$b_{1}$

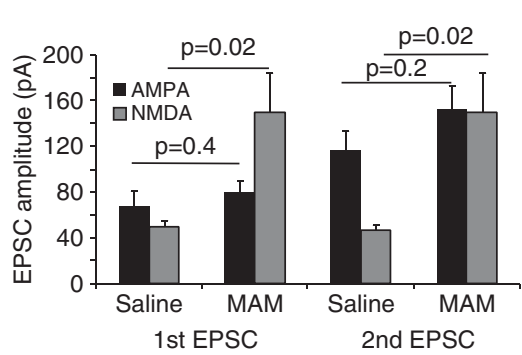

$\mathbf{b}_{2}$
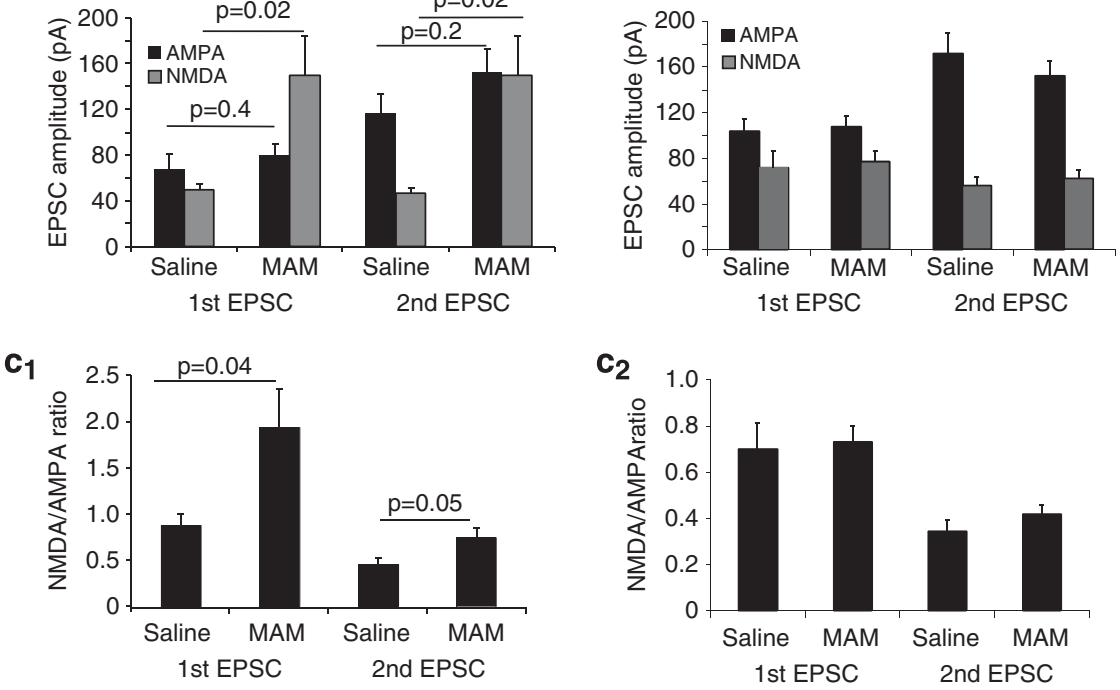

Figure 3 NMDA-EPSC amplitudes are increased in juvenile MAM-exposed rats, but normalized by adolescence. $\left(a_{1}\right.$ and $\left.a_{2}\right)$ Representative paired-pulse traces of stratum radiatum-evoked EPSCs at -60 and $+60 \mathrm{mV}$ from CAI cells of a juvenile $\left(a_{1}\right)$ and $\left(a_{2}\right)$ adolescent saline and MAM-exposed animal. (b, and $b_{2}$ ) Summary histograms show that NMDA-EPSC amplitude is significantly increased in cells from juvenile MAM-exposed animals (saline, $n=7$, MAM $n=9, p=0.02$ and 0.02, respectively), while AMPA-EPSC amplitude is not changed (saline, $n=7$, MAM $n=9, p=0.40$ and 0.42 , respectively). In contrast, both NMDA-EPSC and AMPA-EPSC are not different between adolescent saline and MAM animals (saline, $n=7, M A M n=8, p=0.7$ and 0.6 , respectively). ( $c_{1}$ and $c_{2}$ ) Summary histograms show a significantly increased first NMDA/AMPA ratio in cells from juvenile (saline, $n=7, M A M n=9$, $p=0.04$ ), but not in neurons from adolescent MAM-exposed animals (saline, $n=7, \operatorname{MAM} n=8, p=0.8$ ).

NMDAR function or whether AMPAR function was affected as well, especially given the altered paired-pulse ratio found in adolescence. To determine whether MAM exposure had an effect on functional AMPARs and/or there were changes to presynaptic function, we recorded sEPSC and mEPSC while holding the cell at $-70 \mathrm{mV}$ in juvenile and adolescent animals (Figure $4 a_{1}$ and $b_{1}$ ). We found no differences in either the frequency or amplitude of the sEPSCs or mEPSCs in juvenile (P12-20) MAM- and saline-exposed rats (Juvenile, Figure $4 a_{1}$ and $b_{1}$, sEPSCs: frequency, $\mathrm{Hz}$, saline $0.49 \pm 0.11$, MAM $0.55 \pm 0.122, p=0.74$; amplitude, $\mathrm{pA}$, saline $11.2 \pm 1.62$, MAM $12.7 \pm 1.34, p=0.48 ; n=7$ and 10 , respectively. mEPSCS: frequency, $\mathrm{Hz}$, saline $0.27 \pm 0.05$, MAM $0.24 \pm 0.03, p=0.55$; amplitude, $\mathrm{pA}$, saline $10.6 \pm 1.55$, MAM $9.19 \pm 0.53, p=0.33 ; n=6$ and 8 , respectively). Similarly, we recorded sEPSCs and mEPSCs to assess functional AMPARs and presynaptic function in adolescent (P29-39) rats (Figure $4 a_{2}$ and $b_{2}$ ). However, we found no difference in the frequency or amplitude of sEPSCs or mEPSCs in adolescent saline and MAM animals (adolescent, Figure $4 \mathrm{a}_{2}$ sEPSCs, frequency, $\mathrm{Hz}$, saline $0.549 \pm 0.08$, MAM $0.56 \pm 0.08, p=0.9$; amplitude, $\mathrm{pA}$, saline $9.96 \pm 0.77$, MAM $9.06 \pm 1.2, p=0.5 ; n=7$ and 10 , respectively; mEPSCs, Figure $4 \mathrm{~b}_{2}$, frequency, $\mathrm{Hz}$, saline $0.35 \pm 0.06$, MAM $0.34 \pm$ $0.05, p=0.7$; amplitude, $\mathrm{pA}$, saline $9.3 \pm 0.39$, MAM $8.8 \pm$ $0.91, p=0.8, n=6$ and 7 , respectively). Therefore, MAM exposure selectively and transiently affects NMDAR function in juvenile hippocampal neurons.

\section{Long-Term Potentiation is Impaired in Juvenile MAM-Exposed Rats}

SZ is associated with cognitive deficits, which includes learning and memory processes. For example, it was recently demonstrated that visual plasticity is disrupted in SZ patients (Cavus et al, 2012). In rodents, long-term potentiation is considered to be the cellular correlate for learning and memory, and requires NMDAR function (Lee and Silva, 2009). Because NMDAR function is disrupted in juveniles by MAM exposure, we compared LTP in saline and MAM animals measured in CA1 extracellular field potential recordings after high-frequency $(100 \mathrm{~Hz}, 100$ pulses) Schaffer collateral stimulation (Figure 5a). Interestingly, as exhibited in Figure 5, slices from MAM animals showed a significant reduction in both short-term potentiation (the first 2-min post-tetanic potentiation) and the magnitude of LTP (Figure $5 b$ and c, normalized EPSP slope, average first 2 -min post-tetanus, saline $2.20 \pm 0.23$, MAM $1.54 \pm 0.13, p=0.02$; average last 5 -min post-tetanus, saline $1.77 \pm 0.26$, MAM $1.27 \pm 0.06, p=0.03, n=6$ and 12 , respectively). This may indicate that juvenile MAM animals have learning and memory deficits. We did not test LTP in 

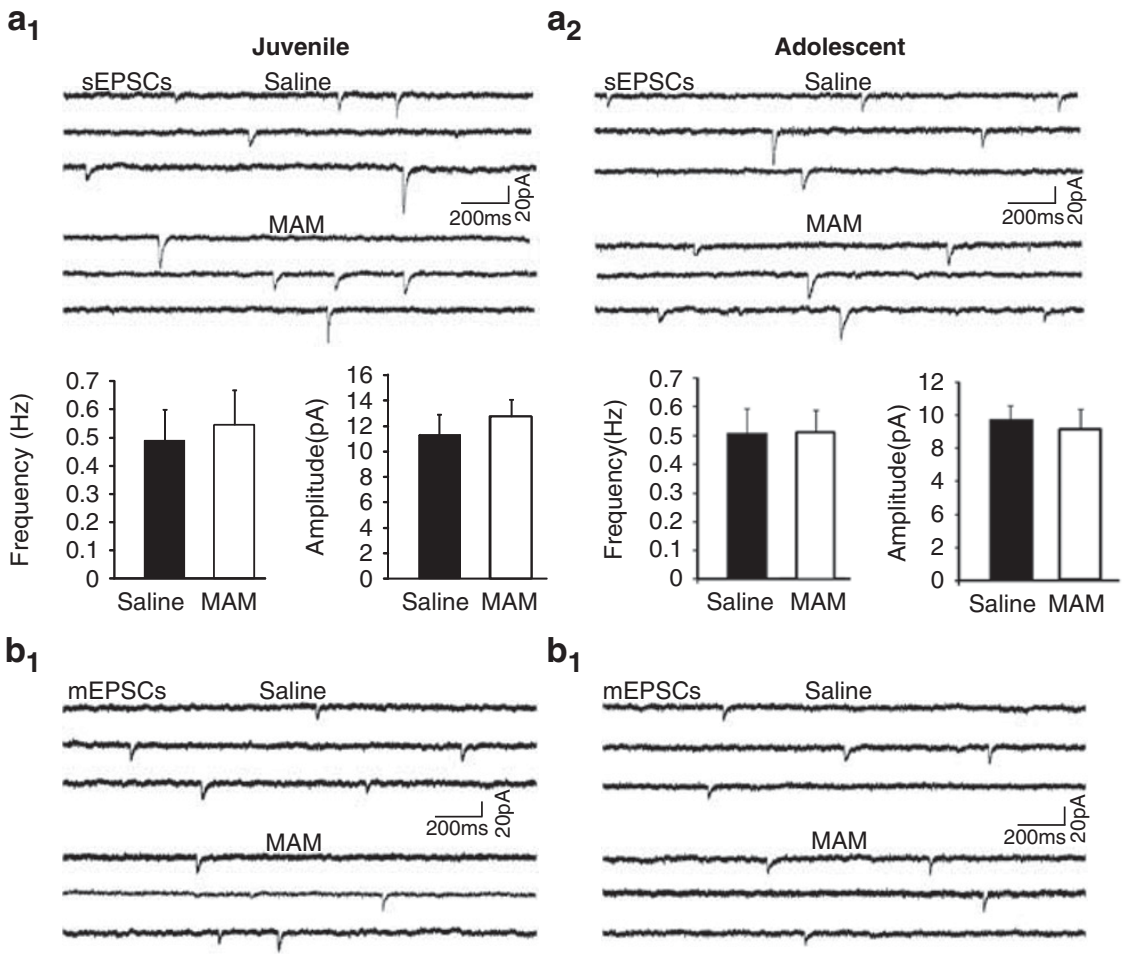

$\mathbf{b}_{1}$
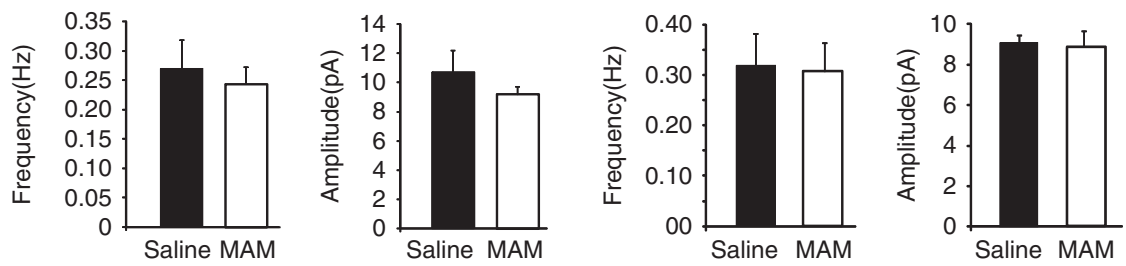

Figure 4 AMPA receptor-mediated currents are not affected by MAM in either juveniles or adolescents. ( $a_{1}$ and $\left.a_{2}\right)$ Sample traces (upper panels) and summary histograms (lower panels) of sEPSCs recorded at $-70 \mathrm{mV}$ in CAI pyramidal neurons from juvenile $\left(a_{1}\right)$ adolescent $\left(a_{2}\right)$ saline and MAM-exposed animals. Summary graphs show no change in sEPSC frequency or amplitude with MAM exposure for juvenile $(p=0.74,0.48$, respectively, saline $n=7$, MAM $n=8$ ) or adolescent animals ( $p=0.9,0.5$, respectively, saline, $n=7$, MAM $n=9)$. ( $b_{1}$ and $b_{2}$ ) Sample traces (upper panels) and summary histograms (lower panels) of mEPSCs recorded at $-70 \mathrm{mV}$ in the presence of TTX in CAI pyramidal neurons from juvenile $\left(b_{1}\right)$ and adolescent (b $\left.b_{2}\right)$ saline- and $M A M-$ exposed animals. MAM exposure did not alter the frequency or amplitude of mEPSCs for either age group (juveniles, $p=0.55,0.33$, respectively, saline $n=6$, MAM, $n=9$; adolescent, $p=0.7,0.8$, respectively, saline $n=6$, MAM $n=7$ ). These data indicate that there is no change in functional AMPARs at synapses in juvenile or adolescent MAM-exposed animals.

adolescence because both AMPA and NMDA functions appear to be mostly normal. Furthermore, it was previously demonstrated that MAM exposure did not affect the induction and magnitude of LTP in adult animals, although there was a substantial deficit in the reversal of LTP, and depotentiation (Sanderson et al, 2012).

\section{MAM Exposure Impairs Spatial Learning in Juvenile and Adolescent Rats}

Previous studies indicate that changes in NMDARs, as well as deficits in long-term potentiation, affect spatial learning and memory (Roberts et al, 2009; Silva et al, 1996; Tang et al, 1999). Furthermore, SZ patients show deficits in performance on a virtual MWM task (Folley et al, 2010). We therefore examined whether the alterations in NMDAR function and long-term potentiation we observed would have behavioral consequences for juvenile MAM animals. We trained juvenile (P17 on training day 1 ) saline and MAM animals on the fixed platform MWM and examined the latency for locating the hidden escape platform. As shown in Figure 6, saline animals learned to find the hidden escape platform across training days. In contrast, in MAM animals the escape latencies were stable without clear changes over training days. By repeated measures ANOVA, there was a significant effect of day $(\mathrm{F}=9.49, p=0.001)$, treatment $(\mathrm{F}=20.04, p=0.002)$, as well as an interaction $(\mathrm{F}=2.73$, $p=0.034$; Figure 6a1, $n=11$ for each group). Post-hoc analysis revealed a significant difference in escape latencies on training days 3,4 , and 5 between saline and MAM animals (Figure 6a1, all $p<0.05$ ).

After training, we tested memory for the platform location with a probe trial. The hidden platform is removed from the MWM and the amount of time the animal spends swimming in the quadrant of the previous platform location is recorded. Animals with a strong memory for the platform location will spend more time swimming in the quadrant where the platform used to be. Not surprising given their 
a

\section{Baseline}

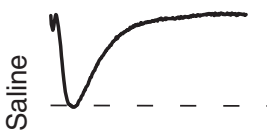

First 2 min post-tetanus

Last 5 min post-tetanus

$$
\text { लำ }
$$
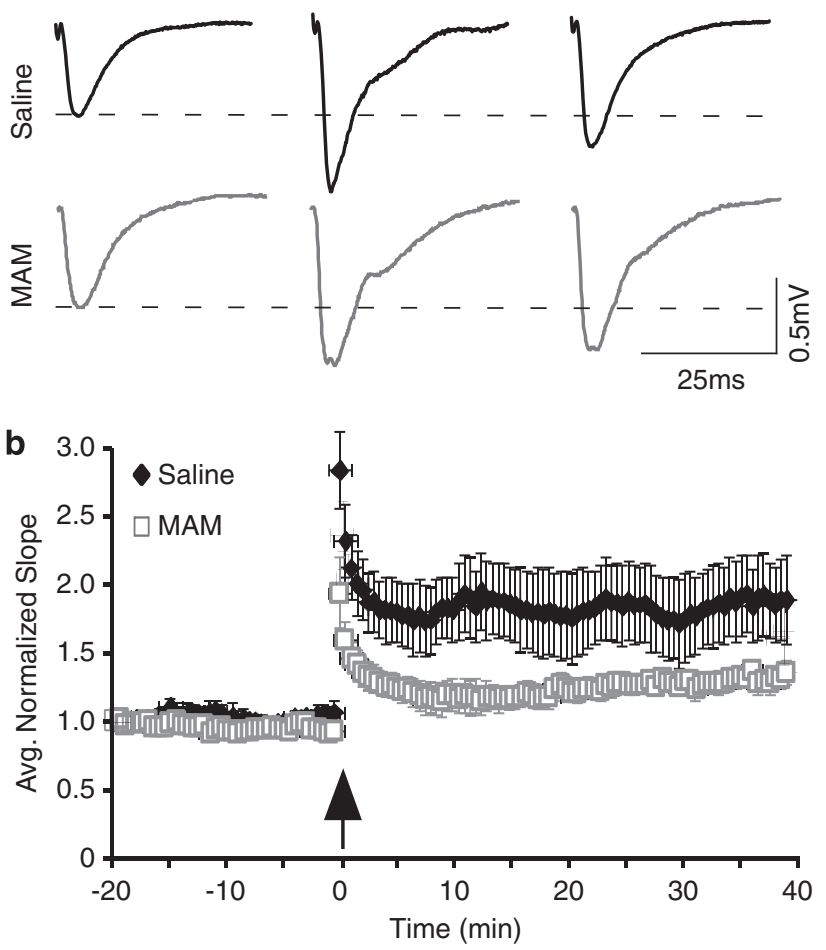

C

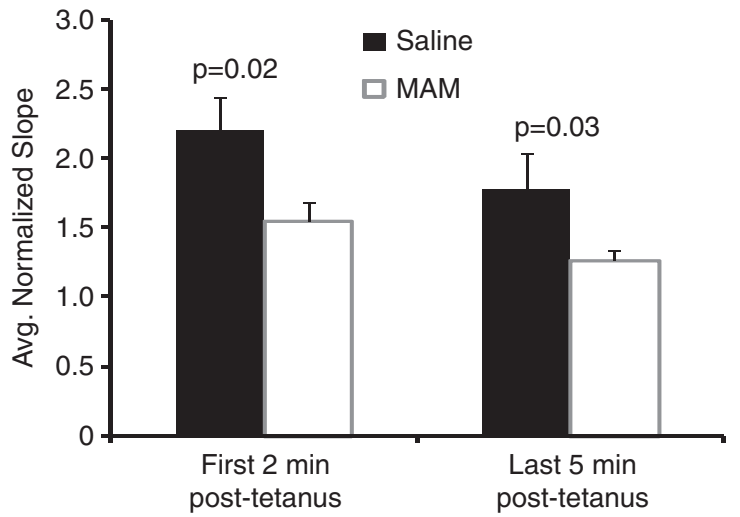

Figure 5 LTP is impaired in slices from juvenile MAM-exposed animals. (a) Representative fEPSPs recorded in the CAI region of the hippocampus evoked by stimulation of Schaffer collaterals during baseline recording and following high-frequency stimulation. Recordings were conducted in slices from animals aged PI5-2I. (b) Summary graph shows the averagenormalized slope of fEPSPs in saline and MAM animals during baseline recording and following high-frequency stimulation. (c) Summary histogram shows that both post-tetanic potentiation and LTP are significantly attenuated in MAM-exposed animals (first 2-min post-tetanus, $p=0.02$; average last 5 -min post-tetanus, $p=0.028$, saline $n=6$ and MAM $n=12$.

poor performance during training, the MAM animals spent significantly less time swimming in the previous target quadrant (Figure $6 \mathrm{a}_{2}, p=0.01$ ). Additionally, MAM animals displayed thigmotaxis, spending a significantly longer amount of time swimming along the edge of the pool (in seconds, saline, $28.2 \pm 1.4$, MAM, $43.6 \pm 2.1, p=0.0002$ ). While there was no difference in swim speeds between juvenile saline and MAM animals (in $\mathrm{cm} / \mathrm{s}$, saline, $23.6 \pm 0.62$, MAM,
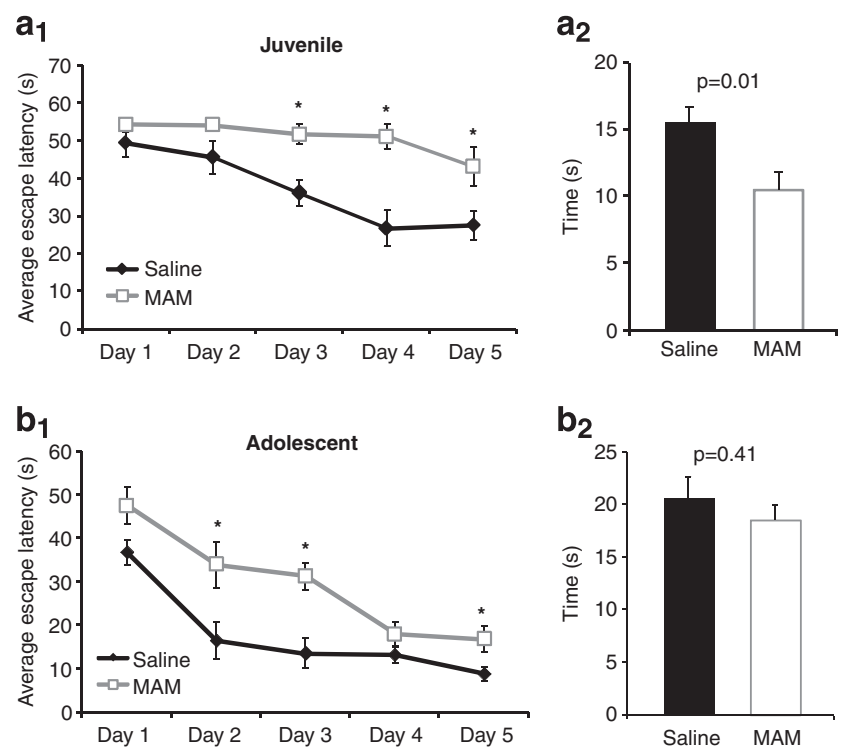

Figure 6 Both juvenile and adolescent MAM animals exhibit learning deficits in the Morris Water Maze. $\left(a_{1}\right.$ and $\left.b_{1}\right)$ Summary line graphs show the average latencies (s) to the escape platform across training on the Morris Water Maze for juvenile $\left(a_{1}\right)$ and adolescent $\left(b_{1}\right)$ saline and MAM animals. Training began on PI7 for juveniles and P40 for adolescents. $\left(a_{1}\right)$ Juveniles exhibited learning deficits with a significant effect of day $(F=9.49$, $p=0.00 \mathrm{I})$, treatment $(F=20.04, p=0.002)$, and an interaction $(F=2.73$, $p=0.034$ ) by repeated measures ANOVA ( $n=1 \mid$ for each group). Posthoc analysis revealed a significant difference in escape latencies on training days 3,4 , and 5 between saline and MAM animals $(* p<0.05)$. $\left(b_{1}\right)$ Although adolescent MAM animals learned the task better than juvenile MAM animals, they still exhibited certain level of learning deficits, with a significant effect of treatment ANOVA ( $F=23.1, p=0.02)$ and an effect of day $(F=24.2, p=0.000 \mathrm{I})$, but no interaction $(F=1.52, p=0.56)$ by repeated measures ANOVA ( $n=7$ for each group). Post-hoc analysis revealed a significant difference in escape latencies on training days 2,3 , and 5 for MAM animals compared with saline $(* p<0.05)$. $\left(a_{2}\right.$ and $\left.b_{2}\right)$ Summary histograms show the time spent in searching the target quadrant during a probe trial for juvenile $\left(a_{2}\right)$ and adolescent $\left(b_{2}\right)$ animals, respectively. $\left(a_{2}\right)$ During a probe trial conducted post-training, juvenile MAM-exposed animals spent significantly less time swimming in the previous target quadrant, indicating low memory for the previous platform location $(p=0.0 \mathrm{I}, n=1 \mathrm{I}) .\left(b_{2}\right)$ In contrast, there was not a significant difference in the amount of time adolescent MAM animals spent swimming in the target quadrant compared to saline animals $(p=0.41, n=7)$. This indicates that adolescent MAM animals have a memory for the previous platform location.

$24.7 \pm 3.5, p=0.74)$, MAM animals had an increased latency to reach the platform during the visible platform trials that we completed following the probe trial (in seconds, saline, $14.1 \pm 2.2$, MAM, $26.4 \pm 4.2, p=0.02$ ). This may reflect a reduced motivation for MAM animals to escape the water. Therefore, reduced NR3A levels, increased NMDAR current, and reduced long-term potentiation is strongly correlated with deficits in spatial learning and memory for juvenile MAM animals.

We next determined whether these behavioral deficits would persist in adolescent MAM animals. We trained adolescent saline and MAM animals (aged P40 on training day 1) on the fixed platform MWM. In contrast to the juveniles, adolescent MAM animals were able to learn the water maze task. However, they still exhibited some deficits, with a significant effect of treatment ANOVA $(\mathrm{F}=23.1$, $p=0.02)$, and an effect of day $(\mathrm{F}=24.2, p=0.0001)$, but no 
interaction $(\mathrm{F}=1.52, p=0.56)$ by repeated measures. Posthoc analysis revealed a significant difference in escape latencies on training days 2,3 , and 5 for MAM animals compared with saline (Figure $6 \mathrm{~b}, p<0.05, n=7$, for each group). Interestingly, during the probe trial, there was no difference between saline and MAM animals in the amount of time spent swimming in the previous target quadrant $(p=0.41)$, indicating an improved memory for the platform location. Furthermore, there was no difference in swim speeds (in cm/s, Saline, $24.7 \pm 1.1$, MAM, $26.1 \pm 1.3, p=0.44$ ) or in the latency to reach a visible platform (in seconds, saline, $11.0 \pm 2.0$, MAM, $11.1 \pm 2.3, p=0.95)$, indicating normal locomotor activity (Flagstad et al, 2004). These results indicate that the adolescent MAM animals, in contrast to their juvenile performance, had an improved memory for the platform location, despite their learning deficits indicated by their longer latencies to find the hidden platform during training. This result is consistent with Hazane et al (2009), who reported severe learning deficits in the MWM for adult MAM-exposed animals. Therefore, recovery in NMDAR protein levels and function in the adolescent appears to be correlated with improved memory performance. However, the persistent learning deficits on the MWM for MAM animals may be the result of decreased NR2B protein levels or a yet unexplored mechanism.

\section{DISCUSSION}

The MAM SZ model has been extensively validated in previous studies (Featherstone et al, 2009; Flagstad et al, 2004; Goto and Grace, 2006; Lodge and Grace, 2007; Moore et al, 2006). However, the majority of work focused on the adult phenotype with only a few behavioral experiments conducted in pre-puberty (Hazane et al, 2009; Le Pen et al, 2006; Moore et al, 2006). For example, most behavioral deficits such as enhanced sensitivity to MK801 and AMPH, as well as impaired pre-pulse inhibition emerge postpuberty (Le Pen et al, 2006; Moore et al, 2006), but deficits in social interaction and spontaneous alternation were reported in pre-pubertal MAM animals (Hazane et al, 2009; Le Pen et al, 2006). Our study is unique in that it is the first to examine early developmental changes to the glutamatergic system that could alter hippocampal development and contribute to the adult MAM phenotype. We initially focused on NMDARs because of their known importance for learning and memory and diseased states, including SZ (Lau and Zukin, 2007). We investigated the effects of prenatal MAM exposure on NMDAR expression and function during early development in the hippocampus. We found that in juvenile MAM rats, NR3A protein levels were transiently decreased which enhanced NMDAR function, but with no effects on AMPAR function. In addition, MAM animals had significantly reduced LTP and impaired performance on the MWM in juveniles. Interestingly for MAMadolescent animals, memory for the task was normal but learning deficits persisted, which may be related to the reduced NR2B protein levels at this developmental time point.

An important finding in this study is that prenatal MAM animals had decreased NR3A proteins levels and an increased NMDAR function in juveniles. NR3A subunits are negative regulators of NMDAR channel function. They confer reduced calcium permeability, lower $\mathrm{Mg}^{2+}$ sensitivity, and decreased channel conductance (Das et al, 1998; Perez-Otano et al, 2001; Sasaki et al, 2002; Tong et al, 2008). Therefore, the increased NMDA-EPSC amplitude in CA1 pyramidal neurons in the MAM-exposed rats is likely the result of reduced NR3A subunits. Furthermore, we observed an increased NMDA/AMPA ratio with no change in AMPAR function in juvenile MAM animals, consistent with those reported in NR3A KO mice (Tong et al, 2008). Presynaptic NR3A-containing NMDARs have been shown in neocortex to alter neurotransmitter release (Larsen et al, 2011). It is therefore possible that MAM exposure alters presynaptic NMDAR function that could influence release probability. Yet, we saw no changes in paired-pulse ratios or the frequencies of both AMPA-sEPSCs and AMPA-mEPSCs, suggesting that there is no change in presynaptic function in juvenile MAM animals, in agreement with a recent study (Sanderson et al, 2012).

Interestingly, the changes in NR3A expression and NMDA-EPSC amplitude were transient. Both NR3A subunit levels and NMDAR function in MAM animals are similar to controls in adolescence. But instead, NR2B protein levels are significantly decreased at this age. The altered NMDAR subunit composition in the synapse could have important consequences for neuronal functioning and development. Several recent studies have reported an altered hippocampal function in adult MAM-exposed animals. Lodge and Grace (2007) first reported an increased neuronal activity in the ventral hippocampus that leads to an enhanced activity of dopaminergic neurons in the ventral tegmental area. Furthermore, two recent studies from the same group have exhibited an increased hyperexcitability, a reduced synaptic transmission, and a disrupted depotentiation but intact LTP and LTD induction in the dorsal hippocampus of adult MAM animals (Hradetzky et al, 2012; Sanderson et al, 2012). In contrast, we found that the neuronal excitabilities in juvenile hippocampus were comparable between salineand MAM-treated animals. This discrepancy is likely attributable to the age difference or recording location, ie, ventral $v s$ dorsal hippocampus. Despite the NMDAR function change, the hippocampal circuit appears relatively intact in the juvenile animals, likely because the deficit in inhibitory transmission appears only after late adolescent (Trantham-Davidson, HL et al, personal communication), whereas the hyperexcitability is probably derived from deficits in synaptic inhibition (Lodge et al, 2009; Sanderson et al, 2012). While these other experiments are all conducted in adult animals, they lend credence to the idea that the early functional changes in NMDARs as we observed in this study could contribute to the malformation of the hippocampal circuit.

Indeed, increasing evidence has shown that altering NMDAR subunit composition and function can affect both brain development and cognitive functioning (Brigman et al, 2010; Gambrill and Barria, 2011; Gray et al, 2011; Tang et al, 1999; von Engelhardt et al, 2008; Yashiro and Philpot, 2008; Zhao et al, 2005). It is generally believed that NMDAR subunit changes such as the NR2B-to-NR2A (Dumas, 2005; Gambrill and Barria, 2011) and the NR3A-to-NR3B switch (Henson et al, 2010; Henson et al, 2008) are important for proper hippocampal development. The early changes in 
NMDAR function observed in this study correspond to a critical time period of extensive hippocampal maturation. During this period, the hippocampus undergoes synaptogenesis, neuronal dendritic arborization, synaptic pruning and remodeling, and maturation of circuitry (Crain et al, 1973; Hsia et al, 1998; Pokorny and Yamamoto, 1981; Swann et al, 1991). These processes, at least partially, rely upon synaptic activation and signaling through NMDARs (Stoneham et al, 2010). Evidence suggests that removal of NR3A from NMDARs is critical for synapse maturation and premature removal of NR3ANMDARs could lead to detrimental effects in developing synapses (Perez-Otano et al, 2006; Roberts et al, 2009). Additionally, reduced NR3A in NMDARs could increase calcium influx, which in turn could easily activate intracellular signaling cascades, leading to excitotoxicity or disrupted synaptic function during development (Fink et al, 2003; Okamoto et al, 2007), and consequently hippocampal dysfunction. Furthermore, the reduced NR2B protein levels could also impact circuit maturation (Akashi et al, 2009; Liu et al, 2004; Monyer et al, 1994; Ohno et al, 2010; Sheng et al, 1994; Wang et al, 2011; Wang et al, 2008; Williams et al, 1993) as well as learning processes (Duffy et al, 2008; Valenzuela-Harrington et al, 2007; Wang et al, 2008; Zhao et al, 2005; Zhou et al, 2007).

It should be pointed out that our finding of enhanced NMDAR function in juvenile MAM animals contrasts with the NMDAR hypofunction associated with SZ in adulthood (Coyle, 2006; Dracheva et al, 2001; Javitt, 2004; Moghaddam and Jackson, 2003; Mohn et al, 1999; Olney et al, 1999; Tamminga, 1999). Downregulated NMDAR subunits are usually seen in postmortem tissue from schizophrenics (Akbarian et al, 1996; Gao et al, 2000; Kristiansen et al, 2007) and animal models with disrupted NMDAR function that have SZ-like symptoms (Belforte et al, 2010; GunduzBruce, 2009; Lisman et al, 2008; Xi et al, 2011). However, the majority of these experiments has been conducted on postmortem adult human brains or in adult animals and thus does not exclude the possibility that NMDAR expression and function can be differentially regulated over development. This is indeed evidenced by our findings of a NMDAR hyperfunction in juveniles and reduced NR2B protein levels in the adolescent MAM animals. Therefore, any dysregulation of NMDAR functioning is likely to impact the developmental process and thus affect synaptic maturation and learning and memory (Henson et al, 2010; Roberts et al, 2009; Wang et al, 2011).

\section{MAM Exposure Alters Learning and Memory in Juvenile Animals}

The enhanced NMDAR function occurs at the same developmental time point as deficits in LTP and the MWM in juvenile MAM animals. While it is unclear if and how the changes to NMDAR function are related to those deficits, previous studies have demonstrated the importance of NMDAR function in both LTP and cognitive performance. NMDAR blockade prevents LTP and impairs performance on the MWM (Morris et al, 1986; Riekkinen and Riekkinen, 1997; Tsien et al, 1996). Indeed, we found that that decreased NR3A in juvenile MAM animals were accompanied with deficits in LTP and performance on the MWM, consistent with a recent report (Roberts et al, 2009), in which downregulation of NR3A-containing NMDARs was found to be required for synapse maturation and memory consolidation. However, it is unlikely that the alterations in NMDAR expression and function are the sole mediators of the deficits in the MWM. NR3A levels recovered by adolescence, yet, learning deficits while lessened compared with the juvenile, persist. We found a significant difference in the adolescents for the escape latency throughout training on the MWM. The remaining learning deficits at the later developmental time point could reflect the reduced NR2B levels in adolescent MAM animals, or a malfunctioning of the hippocampus circuit as a whole. While this learning deficit for MAM animals is consistent with data from the work by Hazane et al (2009) who found similar learning deficits in adult female MAM-exposed SpragueDawley animals, it contrasts with Flagstad et al (2005) who only found reversal learning deficits on the MWM in adult male MAM-exposed Wistar rats. The reason for these discrepancies is not clear, but it could be attributable to gender or strain differences.

Additionally, it is known that performance on the water maze task involves not only hippocampus but also other brain regions, including the prefrontal cortex (Cerqueira et al, 2007; Mogensen et al, 1995). Altered prefrontalhippocampal functioning has been found in both human SZ patients and animal models (Henseler et al, 2010; Lipska et al, 2002a; Lipska and Weinberger, 2002b; Sigurdsson et al, 2010; Tseng et al, 2008), including the MAM model (Goto and Grace, 2006). It is therefore possible that the MAM-induced learning deficits in adolescence reflect altered connectivity between the PFC and hippocampus. Further, adult MAM animals have a greater responsivity to stress, which may also contribute to learning deficits in the MWM (Flagstad et al, 2004; Goto and Grace, 2006; Le Pen et al, 2006).

Still, it remains to be tested whether and how the enhanced NMDAR function during the first several postnatal weeks will affect hippocampal maturation and possibly alter connections with other brain regions. Furthermore, it is unlikely that NMDARs are the only proteins altered throughout development by prenatal MAM exposure (Hradetzky et al, 2012), although AMPAR appears to be unaltered. Future studies are needed to determine how functional connectivity between brain regions is altered throughout postnatal development. Understanding when and how the neurodevelopmental trajectory is altered in the MAM and other SZ models may provide mechanistic insight into its cause and progression, as well as generate possible new avenues for therapeutic intervention.

\section{ACKNOWLEDGEMENTS}

We thank Drs Ramesh Raghupathi and Jed Shumsky for the use of equipment and thoughtful discussion. This work is supported by NIH R01MH232395 to W-J Gao.

\section{DISCLOSURE}

We reaffirm that the content of this manuscript is not under consideration for publication elsewhere nor has the 
information been previously published. All of the authors have fulfilled all conditions required for authorship and have approved the resubmission. Institutional review board approval is not required for this project. The authors declare no financial disclosure and conflict of interests.

\section{Author contributions}

W-JG conceived the study, supervised the project, and wrote the manuscript. MAS designed and carried out all experiments and data analysis of western blots and electrophysiological recordings and she wrote the paper. AEA conducted part of the western blot experiments and read the manuscript.

\section{REFERENCES}

Akashi K, Kakizaki T, Kamiya H, Fukaya M, Yamasaki M, Abe M et al (2009). NMDA receptor GluN2B (GluR epsilon 2/NR2B) subunit is crucial for channel function, postsynaptic macromolecular organization, and actin cytoskeleton at hippocampal CA3 synapses. J Neurosci 29: 10869-10882.

Akbarian S, Sucher NJ, Bradley D, Tafazzoli A, Trinh D, Hetrick WP et al (1996). Selective alterations in gene expression for NMDA receptor subunits in prefrontal cortex of schizophrenics. J Neurosci 16: 19-30.

Belforte JE, Zsiros V, Sklar ER, Jiang Z, Yu G, Li Y et al (2010). Postnatal NMDA receptor ablation in corticolimbic interneurons confers schizophrenia-like phenotypes. Nat Neurosci 13: 76-83.

Bogerts B, Ashtari M, Degreef G, Alvir JM, Bilder RM, Lieberman JA (1990). Reduced temporal limbic structure volumes on magnetic resonance images in first episode schizophrenia. Psychiatry Res 35: 1-13.

Brigman JL, Wright T, Talani G, Prasad-Mulcare S, Jinde S, Seabold GK et al (2010). Loss of GluN2B-containing NMDA Receptors in CA1 hippocampus and cortex impairs long-term depression, reduces dendritic spine density, and disrupts learning. J Neurosci 30: 4590-4600.

Cavus I, Reinhart RM, Roach BJ, Gueorguieva R, Teyler TJ, Clapp WC et al (2012). Impaired visual cortical plasticity in schizophrenia. Biol Psychiatry 71: 512-520.

Cerqueira JJ, Mailliet F, Almeida OF, Jay TM, Sousa N (2007). The prefrontal cortex as a key target of the maladaptive response to stress. J Neurosci 27: 2781-2787.

Coyle JT (2006). Glutamate and schizophrenia: beyond the dopamine hypothesis. Cell Mol Neurobiol 26: 365-384.

Crain B, Cotman C, Taylor D, Lynch G (1973). A quantitative electron microscopic study of synaptogenesis in the dentate gyrus of the rat. Brain Res 63: 195-204.

Cull-Candy S, Brickley S, Farrant M (2001). NMDA receptor subunits: diversity, development and disease. Curr Opin Neurobiol 11: 327-335.

Das S, Sasaki YF, Rothe T, Premkumar LS, Takasu M, Crandall JE et al (1998). Increased NMDA current and spine density in mice lacking the NMDA receptor subunit NR3A. Nature 393: 377-381.

Dracheva S, Marras SAE, Elhakem SL, Kramer FR, Davis KL, Haroutunian V (2001). N-methyl-D-aspartic acid receptor expression in the dorsolateral prefrontal cortex of elderly patients with schizophrenia. Am J Psychiatry 158: 1400-1410.

Duffy S, Labrie V, Roder JC (2008). D-Serine augments NMDANR2B receptor-dependent hippocampal long-term depression and spatial reversal learning. Neuropsychopharmacol 33: 1004-1018.

Dumas TC (2005). Developmental regulation of cognitive abilities: modified composition of a molecular switch turns on associative learning. Prog Neurobiol 76: 189-211.
Featherstone RE, Burton CL, Coppa-Hopman R, Rizos Z, Sinyard J, Kapur S et al (2009). Gestational treatment with methylazoxymethanol (MAM) that disrupts hippocampal-dependent memory does not alter behavioural response to cocaine. Pharmacol Biochem Behav 93: 382-390.

Fink CC, Bayer KU, Myers JW, Ferrell JE Jr., Schulman H, Meyer T (2003). Selective regulation of neurite extension and synapse formation by the beta but not the alpha isoform of CaMKII. Neuron 39: 283-297.

Flagstad P, Glenthoj BY, Didriksen M (2005). Cognitive deficits caused by late gestational disruption of neurogenesis in rats: a preclinical model of schizophrenia. Neuropsychopharmacology 30: $250-260$.

Flagstad P, Mork A, Glenthoj BY, van Beek J, Michael-Titus AT, Didriksen M (2004). Disruption of neurogenesis on gestational day 17 in the rat causes behavioral changes relevant to positive and negative schizophrenia symptoms and alters amphetamineinduced dopamine release in nucleus accumbens. Neuropsychopharmacology 29: 2052-2064.

Folley BS, Astur R, Jagannathan K, Calhoun VD, Pearlson GD (2010). Anomalous neural circuit function in schizophrenia during a virtual Morris water task. Neuroimage 49: 3373-3384.

Gambrill AC, Barria A (2011). NMDA receptor subunit composition controls synaptogenesis and synapse stabilization. Proc Natl Acad Sci USA 108: 5855-5860.

Gao XM, Sakai K, Roberts RC, Conley RR, Dean B, Tamminga CA (2000). Ionotropic glutamate receptors and expression of $\mathrm{N}$ methyl-D-aspartate receptor subunits in subregions of human hippocampus: effects of schizophrenia. Am J Psychiatry 157: 1141-1149.

Goto Y, Grace AA (2006). Alterations in medial prefrontal cortical activity and plasticity in rats with disruption of cortical development. Biol Psychiatry 60: 1259-1267.

Gourevitch R, Rocher C, Le Pen G, Krebs MO, Jay TM (2004). Working memory deficits in adult rats after prenatal disruption of neurogenesis. Behav Pharmacol 15: 287-292.

Grace AA (2012). Dopamine system dysregulation by the hippocampus: implications for the pathophysiology and treatment of schizophrenia. Neuropharmacology 62: 1342-1348.

Gray JA, Shi Y, Usui H, During Matthew J, Sakimura K, Nicoll Roger A (2011). Distinct modes of AMPA receptor suppression at developing synapses by GluN2A and GluN2B: single-cell NMDA receptor subunit deletion in vivo. Neuron 71: 1085-1101.

Gunduz-Bruce H (2009). The acute effects of NMDA antagonism: from the rodent to the human brain. Brain Res Rev 60: 279-286.

Harrison PJ (2004). The hippocampus in schizophrenia: a review of the neuropathological evidence and its pathophysiological implications. Psychopharmacology (Berl) 174: 151-162.

Hazane F, Krebs MO, Jay TM, Le Pen G (2009). Behavioral perturbations after prenatal neurogenesis disturbance in female rat. Neurotox Res 15: 311-320.

Henseler I, Falkai P, Gruber O (2010). Disturbed functional connectivity within brain networks subserving domain-specific subcomponents of working memory in schizophrenia: relation to performance and clinical symptoms. J Psychiatr Res 44: 364-372.

Henson MA, Roberts AC, Perez-Otano I, Philpot BD (2010). Influence of the NR3A subunit on NMDA receptor functions. Prog Neurobiol 91: 23-37.

Henson MA, Roberts AC, Salimi K, Vadlamudi S, Hamer RM, Gilmore JH et al (2008). Developmental regulation of the NMDA receptor subunits, NR3A and NR1, in human prefrontal cortex. Cereb Cortex 18: 2560-2573.

Hradetzky E, Sanderson TM, Tsang TM, Sherwood JL, Fitzjohn SM, Lakics V et al (2012). The Methylazoxymethanol acetate (MAM-E17) rat model: molecular and functional effects in the hippocampus. Neuropsychopharmacology 37: 364-377.

Hsia AY, Malenka RC, Nicoll RA (1998). Development of excitatory circuitry in the hippocampus. J Neurophysiol 79: 2013-2024. 
Javitt DC (2004). Glutamate as a therapeutic target in psychiatric disorders. Mol Psychiatry 9: 984-997.

Kantrowitz JT, Javitt DC (2010). N-methyl-D-aspartate (NMDA) receptor dysfunction or dysregulation: the final common pathway on the road to schizophrenia? Brain Res Bull 83: 108-121.

Kristiansen LV, Huerta I, Beneyto M, Meador-Woodruff JH (2007). NMDA receptors and schizophrenia. Curr Opin Pharmacol 7: 48-55.

Larsen RS, Corlew RJ, Henson MA, Roberts AC, Mishina M, Watanabe $\mathrm{M}$ et al (2011). NR3A-containing NMDARs promote neurotransmitter release and spike timing-dependent plasticity. Nat Neurosci 14: 338-344.

Lau CG, Zukin RS (2007). NMDA receptor trafficking in synaptic plasticity and neuropsychiatric disorders. Nat Rev Neurosci 8: 413-426.

Le Pen G, Gourevitch R, Hazane F, Hoareau C, Jay TM, Krebs MO (2006). Peri-pubertal maturation after developmental disturbance: a model for psychosis onset in the rat. Neuroscience 143: 395-405.

Lee Y-S, Silva AJ (2009). The molecular and cellular biology of enhanced cognition. Nat Rev Neurosci 10: 126-140.

Lewis DA, Levitt P (2002). Schizophrenia as a disorder of neurodevelopment. Annu Rev Neurosci 25: 409-432.

Lipska BK, Aultman JM, Verma A, Weinberger DR, Moghaddam B (2002a). Neonatal damage of the ventral hippocampus impairs working memory in the rat. Neuropsychopharmacology 27: 47-54.

Lipska BK, Weinberger DR (2002b). A neurodevelopmental model of schizophrenia: neonatal disconnection of the hippocampus. Neurotox Res 4: 469-475.

Lisman JE, Coyle JT, Green RW, Javitt DC, Benes FM, Heckers S et al (2008). Circuit-based framework for understanding neurotransmitter and risk gene interactions in schizophrenia. Trends Neurosci 31: 234-242.

Liu XB, Murray KD, Jones EG (2004). Switching of NMDA receptor $2 \mathrm{~A}$ and $2 \mathrm{~B}$ subunits at thalamic and cortical synapses during early postnatal development. J Neurosci 24: 8885-8895.

Lodge DJ, Behrens MM, Grace AA (2009). A loss of parvalbumincontaining interneurons is associated with diminished oscillatory activity in an animal model of schizophrenia. J Neurosci 29: 2344-2354.

Lodge DJ, Grace AA (2007). Aberrant hippocampal activity underlies the dopamine dysregulation in an animal model of schizophrenia. J Neurosci 27: 11424-11430.

Meador-Woodruff JH, Clinton SM, Beneyto M, McCullumsmith RE (2003). Molecular abnormalities of the glutamate synapse in the thalamus in schizophrenia. Ann N Y Acad Sci 1003: 75-93.

Medoff DR, Holcomb HH, Lahti AC, Tamminga CA (2001). Probing the human hippocampus using rCBF: contrasts in schizophrenia. Hippocampus 11: 543-550.

Mogensen J, Wortwein G, Gustafson B, Ermens P (1995). Lnitroarginine reduces hippocampal mediation of place learning in the rat. Neurobiol Learn Mem 64: 17-24.

Moghaddam B, Jackson ME (2003). Glutamatergic animal models of schizophrenia. Ann N Y Acad Sci 1003: 131-137.

Mohn AR, Gainetdinov RR, Caron MG, Koller BH (1999). Mice with reduced NMDA receptor expression display behaviors related to schizophrenia. Cell 98: 427-436.

Monyer H, Burnashev N, Laurie DJ, Sakmann B, Seeburg PH (1994). Developmental and regional expression in the rat brain and functional properties of four NMDA receptors. Neuron 12: 529-540.

Moore H, Jentsch JD, Ghajarnia M, Geyer MA, Grace AA (2006). A neurobehavioral systems analysis of adult rats exposed to methylazoxymethanol acetate on E17: implications for the neuropathology of schizophrenia. Biol Psychiatry 60: 253-264.

Morris RG, Anderson E, Lynch GS, Baudry M (1986). Selective impairment of learning and blockade of long-term potentiation by an $N$-methyl-D-aspartate receptor antagonist, AP5. Nature 319: $774-776$.
Ohno T, Maeda H, Murabe N, Kamiyama T, Yoshioka N, Mishina $M$ et al (2010). Specific involvement of postsynaptic GluN2Bcontaining NMDA receptors in the developmental elimination of corticospinal synapses. Proc Natl Acad Sci USA 107: $15252-15257$.

Okamoto K-I, Narayanan R, Lee SH, Murata K, Hayashi Y (2007). The role of CaMKII as an F-actin-bundling protein crucial for maintenance of dendritic spine structure. PNAS 104: 6418-6423.

Olney JW, Newcomer JW, Farber NB (1999). NMDA receptor hypofunction model of schizophrenia. J Psychiatr Res 33: 523-533.

Perez-Otano I, Lujan R, Tavalin SJ, Plomann M, Modregger J, Liu X-B et al (2006). Endocytosis and synaptic removal of NR3A-containing NMDA receptors by PACSIN1/syndapin1. Nat Neurosci 9: 611-621.

Perez-Otano I, Schulteis CT, Contractor A, Lipton SA, Trimmer JS, Sucher NJ et al (2001). Assembly with the NR1 subunit is required for surface expression of NR3A-containing NMDA receptors. J Neurosci 21: 1228-1237.

Pokorny J, Yamamoto T (1981). Postnatal ontogenesis of hippocampal CA1 area in rats. I. Development of dendritic arborisation in pyramidal neurons. Brain Res Bull 7: 113-120.

Riekkinen M, Riekkinen P Jr. (1997). Dorsal hippocampal muscarinic acetylcholine and NMDA receptors disrupt water maze navigation. Neuroreport 8: 645-648.

Roberts AC, Díez-García J, Rodriguiz RM, López IP, Luján R, Martínez-Turrillas $\mathrm{R}$ et al (2009). Downregulation of NR3Acontaining NMDARs is required for synapse maturation and memory consolidation. Neuron 63: 342-356.

Sanderson TM, Cotel MC, O’Neill MJ, Tricklebank MD, Collingridge GL, Sher E (2012). Alterations in hippocampal excitability, synaptic transmission and synaptic plasticity in a neurodevelopmental model of schizophrenia. Neuropharmacology 62: 1349-1358.

Sasaki YF, Rothe T, Premkumar LS, Das S, Cui J, Talantova MV et al (2002). Characterization and comparison of the NR3A subunit of the NMDA receptor in recombinant systems and primary cortical neurons. J Neurophysiol 87: 2052-2063.

Sheng M, Cummings J, Roldan LA, Jan YN, Jan LY (1994). Changing subunit composition of heteromeric NMDA receptors during development of rat cortex. Nature 368: 144-147.

Sigurdsson T, Stark KL, Karayiorgou M, Gogos JA, Gordon JA (2010). Impaired hippocampal-prefrontal synchrony in a genetic mouse model of schizophrenia. Nature 464: 763-767.

Silva AJ, Rosahl TW, Chapman PF, Marowitz Z, Friedman E, Frankland PW et al (1996). Impaired learning in mice with abnormal short-lived plasticity. Curr Biol 6: 1509-1518.

Spear LP (2000). The adolescent brain and age-related behavioral manifestations. Neurosci Biobehav Rev 24: 417-463.

Stoneham ET, Sanders EM, Sanyal M, Dumas TC (2010). Rules of engagement: factors that regulate activity-dependent synaptic plasticity during neural network development. Biol Bull 219: 81-99.

Swann JW, Smith KL, Brady RJ (1991). Age-dependent alterations in the operations of hippocampal neural networks. Ann NY Acad Sci 627: 264-276.

Tamminga C (1999). Glutamatergic aspects of schizophrenia. $\mathrm{Br} J$ Psychiatry Suppl 37: 12-15.

Tang YP, Shimizu E, Dube GR, Rampon C, Kerchner GA, Zhuo M et al (1999). Genetic enhancement of learning and memory in mice. Nature 401: 63-69.

Tong G, Takahashi H, Tu S, Shin Y, Talantova M, Zago W et al (2008). Modulation of NMDA receptor properties and synaptic transmission by the NR3A subunit in mouse hippocampal and cerebrocortical neurons. J Neurophysiol 99: 122-132.

Tseng KY, Lewis BL, Hashimoto T, Sesack SR, Kloc M, Lewis DA et al (2008). A neonatal ventral hippocampal lesion causes functional deficits in adult prefrontal cortical interneurons. J Neurosci 28: 12691-12699. 
Tsien JZ, Huerta PT, Tonegawa S (1996). The essential role of hippocampal CA1 NMDA receptor-dependent synaptic plasticity in spatial memory. Cell 87: 1327-1338.

Valenzuela-Harrington M, Gruart A, Delgado-Garcia JM (2007). Contribution of NMDA receptor NR2B subunit to synaptic plasticity during associative learning in behaving rats. Eur $J$ Neurosci 25: 830-836.

von Engelhardt J, Doganci B, Jensen V, Hvalby $\emptyset$, Göngrich C, Taylor A et al (2008). Contribution of hippocampal and extrahippocampal NR2B-containing NMDA receptors to performance on spatial learning tasks. Neuron 60: 846-860.

Wang C-C, Held Richard G, Chang S-C, Yang L, Delpire E, Ghosh A et al (2011). A critical role for GluN2B-containing NMDA receptors in cortical development and function. Neuron 72: 789-805.

Wang HX, Gao WJ (2009). Cell type-specific development of NMDA receptors in the interneurons of rat prefrontal cortex. Neuropsychopharmacol 34: 2028-2040.

Wang HX, Gao WJ (2010). Development of calcium-permeable AMPA receptors and their correlation with NMDA receptors in fast-spiking interneurons of rat prefrontal cortex. J Physiol 588: 2823-2838.

Wang HX, GGr Stradtman, Wang XJ, Gao WJ (2008). A specialized NMDA receptor function in layer 5 recurrent microcircuitry of the adult rat prefrontal cortex. Proc Natl Acad Sci USA 105: 16791-16796.

Williams K, Russell SL, Shen YM, Molinoff PB (1993). Developmental switch in the expression of NMDA receptors occurs in vivo and in vitro. Neuron 10: 267-278.

Witthaus H, Kaufmann C, Bohner G, Ozgurdal S, Gudlowski Y, Gallinat J et al (2009). Gray matter abnormalities in subjects at ultra-high risk for schizophrenia and first-episode schizophrenic patients compared to healthy controls. Psychiatry Res 173: 163-169.

Xi D, Li Y-C, Snyder MA, Gao RY, Adelman AE, Zhang W et al (2011). Group II metabotropic glutamate receptor agonist ameliorates MK-801-induced dysfunction of NMDA receptors via Akt/GSK-3beta pathway. Neuropsychopharmacol 36: 1260-1274.

Yashiro K, Philpot BD (2008). Regulation of NMDA receptor subunit expression and its implications for LTD, LTP, and metaplasticity. Neuropharmacology 55: 1081-1094.

Zhao M-G, Toyoda H, Lee Y-S, Wu L-J, Ko SW, Zhang X-H et al (2005). Roles of NMDA NR2B subtype receptor in prefrontal long-term potentiation and contextual fear memory. Neuron 47: 859-872.

Zhou Y, Takahashi E, Li W, Halt A, Wiltgen B, Ehninger D et al (2007). Interactions between the NR2B receptor and CaMKII modulate synaptic slasticity and spatial learning. J Neurosci 27: 13843-13853. 